\title{
Intravenous immunoglobulin therapy for resistant ocular Behçet's disease
}

\author{
Nir Seider, Itzchak Beiran, Jehudith Scharf, Benjamin Miller
}

\begin{abstract}
Aims-The present report was aimed at finding out whether gammaglobulin could have a role in treating ocular Behçet's disease (BD) refractory to accepted medical therapy.

Methods-Six eyes of four patients with ocular BD refractory to steroids and cyclosporin A were treated with a course of intravenous gammaglobulin and followed up for their response to treatment. Results-All six eyes of all four patients showed good response to gammaglobulin therapy.

Conclusion-Gamma globulin may have a role in treating refractory ocular BD. A wide range of controlled studies with longer follow up is needed to substantiate this impression.

(Br f Ophthalmol 2001;85:1287-1288)
\end{abstract}

Corticosteroids, cytotoxic agents (chlorambucil, cyclophosphamide, and azathioprine), and cyclosporin $\mathrm{A}(\mathrm{Cs} \mathrm{A})$ have been reported to have different rates of success in the treatment of ocular Behçet's disease (BD), yet their side effects limit their long term use. ${ }^{1}$ The present report is a preliminary study on the effect of intravenous immunoglobulin treatment on active ocular BD.

Department of

Ophthalmology,

Rambam Medical

Center, Haifa, Israel

N Seider

I Beiran

J Scharf

B Miller

The Bruce Rappaport

Faculty of Medicine,

Technion-Israel

Institute of

Technology, Haifa,

Israel

J Scharf

B Miller

Correspondence to: Itzchak Beiran, $\mathrm{MD}$

Department of

Ophthalmology, Rambam

Medical Center, POB 9602,

31096 Haifa, Israel

ibeiran@hotmail.com

Accepted for publication 23 May 2001

\section{Patients and methods}

Four patients were included in the study. Clinical details of patients are presented in the Results section.

The study protocol included administration of $0.4 \mathrm{~g} / \mathrm{kg}$ body weight daily of intravenous immunoglobulin (Omr-IgG-am, a 5\% solution of human normal immunoglobulin G; Omrix Biopharmaceuticals Ltd, Nes-Ziona, Israel) five times in the first week, an additional three applications in the first month, once every 20 days for the next 3 months, then every 6 weeks thereafter until the completion of 1 year of treatment. Infusion was given in a 1 hour drip, and the patient was hospitalised for 24 hours post infusion for medical monitoring; no other specific precautions were taken. The treatment protocol was based on a report by Leif Tallstedt (personal communication).
Results

PATIENT 1

A 49 year old woman suffered from BD for 20 years. Eight episodes of panuveitis in the 5 years before her present admission were treated by corticosteroids and CsA, leading to gradual improvement over a 6-12 week course. Upon admission, visual acuity was $3 / 60$ in the right eye and $2 / 60$ in the left eye. The right eye had ciliary injection, keratic precipitate, cells and flare +3 in the anterior chamber, dense posterior subcapsular cataract, +3 cells in the vitreous, normal disc, macular oedema, and sheathing of the retinal arteries in the periphery. The left eye was quiet, with a grade 4 macular hole.

The right eye did not respond to a 2 week course of local hourly and systemic full dose steroid treatment. A course of gammaglobulin was administered according to the protocol. The inflammatory process showed improvement after the first two gammaglobulin applications and resolved to the degree of no cells and +1 flare in 10 days. After 3 months of treatment the right eye underwent cataract surgery, after which the protocol was continued. During 1 year of treatment the eye remained quiet, with $6 / 10$ vision. At the $2 \frac{1}{2}$ year follow up after completion of gammaglobulin treatment, a single attack of panuveitis was observed. This attack was treated by steroids and resolved within 1 month of treatment.
PATIENT 2

A 30 year old woman with BD was seen for first attack of panuveitis in both eyes. Examination revealed $6 / 9$ vision, keratic precipitates in both corneas, +3 cells and flare in both anterior chambers, and +3 cells in the vitreous, as well as macular oedema and sheathing of macular arterioles. The patient did not respond to a 2 week course of full dose systemic corticosteroids and was put on gammaglobulin treatment. Ocular inflammation improved in 5 days, and full resolution was noted in 10 days. The patient was withdrawn from steroid therapy and was treated with only a nonsteroidal anti-inflammatory drug. Over 1 year on the treatment protocol, as well as an additional year's follow up after completion of treatment, vitreal remission was retained, with $6 / 6$ vision in each eye. 
PATIENT 3

A 16 year old boy with $\mathrm{BD}$ presented with monocular panuveitis in the left eye, with $6 / 12$ vision, +3 cells and flare in the anterior chamber, and snowballs in the vitreous. Three weeks of full dose steroids and CsA treatment with therapeutic blood levels did not bring about any improvement. Three weeks after intravenous gammaglobulin was begun by the protocol, the eye improved. After 6 weeks, no sign of active inflammation, with visual acuity of $6 / 6$, was achieved, and the eye was kept in remission for up to 1 year after completion of treatment.

PATIENT 4

A 41 year old woman with BD presented with bilateral panuveitis and vasculitis unimproved with 2 weeks of full dose systemic steroids and CsA (with therapeutic blood levels). In the year preceding the present attack, she had six episodes of panuveitis that resolved after local and systemic full dose steroids and CsA. Visual acuity was $6 / 24$ in the right eye and 3/60 in the left eye. Both eyes showed keratic precipitates and +3 cells and flare in the anterior chamber. Diffuse retinal oedema and +3 cells in the vitreous were observed in both retinas. After 5 weeks of treatment, the inflammation abated and visual acuity improved to $6 / 7$ in the right eye and 6/9 in the left eye. After 6 months of stabilisation, the patient was lost to follow up and treatment. Three months later she returned with a relapse, +2 cells in the anterior chamber and vitreous. The patient was put back on gammaglobulin therapy, and the inflammation abated in 2 weeks, with stabilisation at 1 year under the treatment protocol without steroids. There were no recurrences in the 1 year follow up after completion of treatment.

\section{Discussion}

The use of intravenous immunoglobulin for autoimmune related ocular conditions has been reported for relapsing steroid dependent optic neuritis, ${ }^{2}$ ocular involvement of Wegener's granulomatosis, ${ }^{3}$ ocular cicatricial pemphigoid ${ }^{4}$ and uveitis, ${ }^{56}$ as well as for neurological manifestations of $\mathrm{BD}{ }^{7}$

It seems from our results that the use of an immunoglobulin brought the acute inflammation, uncontrolled by corticosteroids and/or CsA, under control and preserved the remission for a period of at least 1 year. A $2-6 \%$ adverse reaction rate is reported in the literature for immunoglobulin treatment, including a range of symptoms from mild to severe, mainly aseptic meningitis and full hypersensitivity type III reactions. ${ }^{8}$ No such reaction was observed in our series, probably because of small sample size and to slow infusion rate, known to decrease the appearance of side effects. ${ }^{8}$ From our first case it seems that gammaglobulin may have a role in presurgical and postsurgical management of BD patients. The fourth case gave us an inadvertent rechallenge test for the treatment.

The three main shortcomings of our present report are the small number of patients, the lack of controls, and the relatively short follow up. We believe that our results are encouraging enough to justify a wider controlled study with longer follow up on the role of gammaglobulin in ocular BD.

1 Jabs DA. Ocular manifestations of the rheumatic diseasesBehçet disease. In: Tasmin W, Jaeger EA, eds. Duane's clinical ophthalmology. Revised ed. Philadelphia: Lippincott/ Williams and Wilkins, 1998;5:26-9.

2 Frohman LP, Cook SD, Bielory L. Dysgammaglobulinemia in steroid-dependent optic neuritis: response to gammaglobulin treatment. $\mathcal{F}$ Clin Neuroophthalmol 1991;11:241-5.

3 Blum M, Andrassy K, Adler D, et al. Early experience with intravenous immunoglobulin treatment in Wegener's intravenous immunoglobulin treatment in Wegener's Exp Ophthalmol 1997;235:599-602.

4 Bohn J, Benfeldt E, Dabelsteen E, et al. Treatment of ocular cicatricial pemphigoid with intravenous gammaglobulin cicatricial pemphigoid with intravenous ga
(letter). Acta Derm Venereol 1998;78:316-7.

5 Sunakawa M. High-dose intravenous gammaglobulin therapy for uveitis. Metab Pediatr Syst Ophthalmol 1989;12: 93-5.

6 Rosenbaum JT, George RK, Gordon C. The treatment of refractory uveitis with intravenous immunoglobulin. $A m \mathcal{F}$ Ophthalmol 1999;127:545-9.

7 Calzada-Sierra DJ, Garcia-Fidalgo J, Mustelier-Becquer R, et al. Neurobehçet. Presentacion de tres casos. Rev Neurol 1999;28:1166-9.

8 DRUGDEX drug evaluation. September 1999. Immune globulin-adverse effects. 